\title{
LACK OF INHIBITION OF THE ANTI-MALARIAL ACTION OF SULFADOXINE-PYRIMETHAMINE BY FOLIC ACID SUPPLEMENTATION WHEN USED FOR INTERMITTENT PREVENTIVE TREATMENT IN GAMBIAN PRIMIGRAVIDAE
}

\author{
AMADOU MBAYE, KESHENA RICHARDSON, BABA BALAJO, SAM DUNYO, CAROLINE SHULMAN, \\ PAUL MILLIGAN, BRIAN GREENWOOD,* AND GIJS WALRAVEN \\ Medical Research Council Laboratories, Banjul, The Gambia; Department of Health, The Government of The Gambia, Banjul, \\ The Gambia; Department of Infectious and Tropical Diseases, London School of Hygiene and Tropical Medicine, \\ London, United Kingdom
}

\begin{abstract}
Folic acid is frequently given to pregnant women at the same time as intermittent preventive treatment (IPTp) with sulfadoxine/pyrimethamine (SP), but it is not known if it interferes with the anti-malarial activity of SP. To investigate this concern, 1,035 Gambian primigravidae were randomized to receive either folic acid (500-1,500 $\mu \mathrm{g} / \mathrm{day})$ together with oral iron (522) or oral iron alone (513) for 14 days at the same time as they received IPTp with SP. On presentation, 261 women $(25 \%)$ had Plasmodium falciparum asexual parasitemia. Prevalences of parasitemia on day 14 after treatment were similar in both groups: $5.7 \%$ (26 of 458) in the iron plus folic acid group and $4.9 \%(22$ of 446$)$ in the iron alone group (risk difference $=0.74 \%, 95 \%$ confidence interval $[\mathrm{CI}]=-2.2 \%$ to $3.7 \%$ ). Parasitologic cure was observed in $116(91 \%)$ of 128 of women who were parasitemic on presentation and who received iron and folic acid and in $122(92 \%)$ of 133 women who received iron alone (difference $=1.1 \%, 95 \% \mathrm{CI}=-5.6 \%$ to $8.0 \%$ ). Women who received folic acid and iron had a slightly higher mean hemoglobin concentration at day 14 than women who had received iron alone $($ difference $=0.14 \mathrm{~g} / \mathrm{dL}, 95 \% \mathrm{CI}=0.01-0.27 \mathrm{~g} / \mathrm{dL}$ ). The results of this study suggest that in an area of low SP resistance, administration of folic acid to pregnant women in a dose of 500-1,500 $\mu \mathrm{g} / \mathrm{day}$ will not interfere with the protective effect of SP when used for IPTp.
\end{abstract}

\section{INTRODUCTION}

Malaria adversely affects the outcome of pregnancy for both the mother and her baby. ${ }^{1}$ In areas of low transmission, malaria in pregnancy may result in a severe, acute infection and even in the death of the mother. In areas of medium or high transmission, malaria is an important cause of severe anemia in pregnant women and of low birth weight, both of which may reduce infant survival. ${ }^{2,3}$ In areas of medium or high transmission, many malaria infections during pregnancy are asymptomatic and difficult to detect and treat. Thus, the primary public health strategy for controlling malaria in pregnancy is prevention. The two main tools used to prevent malaria in pregnancy are insecticide-treated bed nets ${ }^{4}$ and intermittent preventive treatment (IPTp). The latter, which involves the administration of a full therapeutic dose of an antimalarial drug to pregnant women at fixed times during the course of their pregnancy, regardless of whether they are known to be infected, is easier to deliver than sustained chemoprophylaxis and equally effective. ${ }^{5-8}$ The drug currently recommended for IPTp is sulfadoxine-pyrimethamine (SP), a long-acting folate antagonist. How SP achieves its effect when used for IPTp is uncertain; it is not known if it acts by clearing established infections intermittently or whether its prophylactic effect is more important.

In industrialized countries, folic acid supplementation at a dose of $500 \mu \mathrm{g} /$ day is recommended prior to conception and in the first trimester of pregnancy to prevent neural tube defects, and it is given in higher doses to women who have previously had a baby with a neural tube defect. In developing countries, folic acid supplementation is used widely in the

\footnotetext{
* Address correspondence to Brian Greenwood, Department of Infectious and Tropical Diseases. London School of Hygiene and Tropical Medicine, Keppel Street, London WC1E 7HT, United Kingdom. E-mail: brian.greenwood@lshtm.ac.uk
}

second and third trimesters, together with iron, to prevent nutritional anemia. Folic acid is given to pregnant women attending antenatal clinics across Africa, including those situated in areas where malaria is endemic. Because SP exerts its anti-malarial activity by interfering with the folic acid metabolism of the parasite, concerns have been expressed that folic acid supplementation of pregnant women at antenatal clinics could interfere with the ability of SP to provide protection when used for IPTp because some strains of Plasmodium falciparum can use free folate directly. To investigate this possibility, we have compared the efficacy of SP when used for IPTp in Gambian primigravidae who received folic acid either at the same time as they received IPTp with SP or two weeks later.

\section{MATERIALS AND METHODS}

Study area and population. The study was carried out in 14 mother and child health clinics situated on the north and south banks of the Gambia River near the town of Farafenni, The Gambia in the center of the country. In this area, malaria is highly seasonal with nearly all infections occurring during the rainy season, which lasts from June to November. ${ }^{9}$ The entomologic inoculation rate varies substantially from village to village but is within the range of $10-50$ infectious bites per year. During an 18-month period from July 2002 to December 2003 , primigravidae who attended one of the study clinics were reviewed as to their eligibility to join the study. Eligibility criteria were pregnancy greater than 15 weeks, hemoglobin $(\mathrm{Hb})$ concentration $>7 \mathrm{~g} / \mathrm{dL}$, absence of any serious underlying disease, absence of a history of an adverse response to sulfonamides, and residence in the study area and willingness to be visited at home.

Recruitment and randomization. If a pregnant woman met the study entry criteria, written, informed consent to join the study was obtained and a questionnaire was completed. This 
questionnaire sought information on age, village of residence, education, socioeconomic status, bed net use, past medical and obstetric history, and previous treatment during the pregnancy. A finger prick blood sample was obtained for determination of the $\mathrm{Hb}$ concentration and for preparation of two blood films. Provided that the $\mathrm{Hb}$ concentration was $>7 \mathrm{~g} / \mathrm{dL}$, the woman was given a study number and SP was administered under supervision. The woman was then given the randomization envelope bearing the same study number. This envelope contained iron and folic acid tablets or iron tablets alone. Envelopes were pre-packaged by a person who had no further direct involvement in the trial.

Treatments. All women received directly observed treatment with three tablets of SP (25 mg of pyrimethamine and $500 \mathrm{mg}$ of sulfadoxine) (Cosmos Pharmaceuticals, Nairobi, Kenya). The content and the solubility of the tablets were confirmed by high performance liquid chromatography. Women in the immediate treatment group were then given iron and folic acid tablets $(500 \mu \mathrm{g}$ of folic acid and $47 \mathrm{mg}$ of ferrous sulfate per tablet) to be taken at home once per day for 14 days. Women with an $\mathrm{Hb}$ concentration of $7-9 \mathrm{~g} / \mathrm{dL}$ were asked to take three tablets a day, those with an $\mathrm{Hb}$ concentration of $9-11 \mathrm{~g} / \mathrm{dL}$ were asked to take two tablets a day, and those with an $\mathrm{Hb}$ concentration $\geq 11 \mathrm{~g} / \mathrm{dL}$ were asked to take one tablet a day in line with routine practice in The Gambia. Thus, women in this group received 500-1,500 $\mu \mathrm{g}$ of folic acid per day. Women in the delayed treatment group were given oral iron tablets $(60 \mathrm{mg}$ of ferrous sulfate per tablet) to be taken at home once per day for 14 days, 1-3 tablets according to $\mathrm{Hb}$ concentration as described above. At the end of this period, women in this group were given a supply of iron and folic acid tablets to be taken as described above for women in the immediate treatment group.

Surveillance. Women were visited at home by a field worker 14 days after treatment and a finger prick blood sample was obtained for repeat determination of $\mathrm{Hb}$ concentration and for the preparation of two thick blood films. Any iron or folic acid tablets that had not been used were collected to measure compliance.

Laboratory methods. Hemoglobin concentration was measured with a Hemocue device (Hemocue AB, Ängelholm, Sweden). Thick blood films were stained with Giemsa and read by two microscopists who were blind to the treatment code. If results were discrepant, the slide was read by a third microscopist and the majority view was used for analysis. One hundred high-power fields were read before a slide was declared negative.

Sample size. The sample size was calculated to be able to demonstrate that the efficacy of SP was not impaired by taking folic acid. We anticipated that $5 \%$ of the women in the iron alone group would be parasitemic on day 14 after treatment with SP, and we considered that it would be of clinical importance if folic acid increased this prevalence to $\geq 10 \%$. For a test of non-inferiority with $90 \%$ power, and allowing for loss to follow-up, 450 women were required in each arm. This number gave adequate power to compare treatment failure rates among women who were parasitemic at enrollment, assuming an initial prevalence of parasitemia of $30 \%$ among women enrolled.

Data management and statistical methods. After data had been double entered and verified, the database was locked, archived at the Medical Research Council (MRC) laborato- ries, and a copy was sent to the chairman of the study's Data Safety Monitoring Board (DSMB). Statistical analyses were done using STATA version 8 (Stata Corporation, College Station, TX). The primary trial end point was the prevalence of $P$. falciparum asexual stage parasitemia 14 days after treatment. Confidence intervals (CIs) (95\% and 99\%) were calculated for the difference in parasite prevalence. Since the study was designed as a non-inferiority trial, conventional tests of the hypothesis of no treatment effect were not appropriate. Effects of covariates (clinic, age, gestational age at enrollment, ethnic group, educational level, marital status, and bed net use) were investigated using a generalized linear model. Parasite prevalence at day 14 in women who had $P$. falciparum parasitemia on presentation and an $\mathrm{Hb}$ concentration at day 14 (adjusted for baseline $\mathrm{Hb}$ concentration) were secondary end points.

Ethics. The trial was reviewed and approved by the Gambia Government/MRC and London School of Hygiene and Tropical Medicine ethics committees. The study design was reviewed and approved by a DSMB that monitored the course of the study and approved the analytical plan.

\section{RESULTS}

Trial profile. A total of 1,687 women were screened, of whom 1,035 were recruited and randomized to receive either early (522) or delayed (513) folic acid supplementation (Figure 1). The main reason for initial exclusion from the study

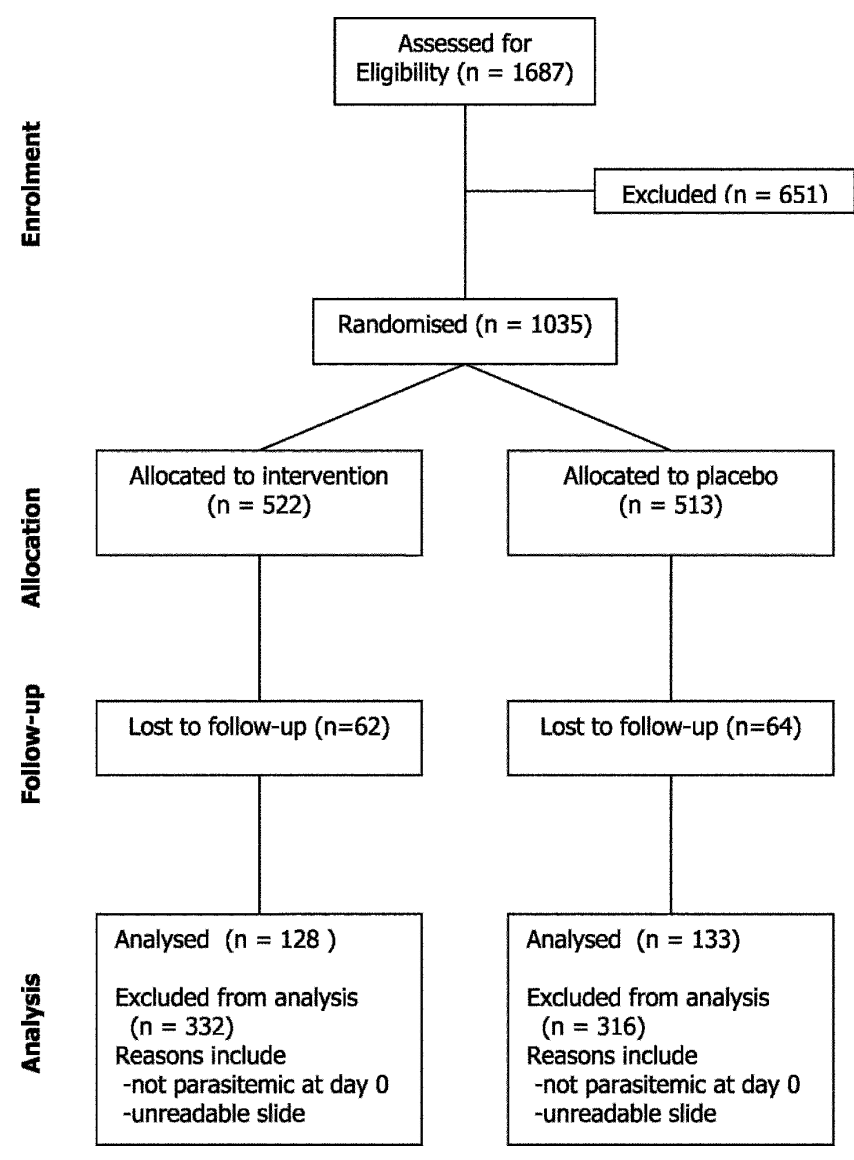

Figure 1. Trial profile. 
was residence in neighboring Senegal. Senegalese women are reluctant to cross the border for repeated clinic visits and it was thought best to exclude them before entry into the trial. Sixty-two women $(12 \%)$ in the early supplementation group and $64(12 \%)$ in the delayed supplementation group were lost to follow-up, mainly because of their travel outside the study area. Comparison of baseline characteristics showed no significant differences between defaulters and those who were seen on day 14. The characteristics of women allocated to each of the study groups are shown in Table 1 . The two treatment groups were similar with respect to baseline characteristics. To check compliance with treatment, blister packs obtained from 794 women were inspected; $71 \%$ had no leftover medication (69\% [282 of 406] in the early supplementation group and $73 \%$ [283 of 388] in the delayed supplementation group).

Response to treatment with SP. Overall, 261 (29\%) of 904 women were parasitemic at enrollment. The prevalence of parasitemia at enrollment increased during the transmission season from $18 \%$ in July to $45 \%$ in December and then decreased during the following dry season. On day 14 after treatment with SP, the prevalence of $P$. falciparum asexual stage parasitemia (regardless of whether parasites were present on day 0$)$ was $26(5.7 \%)$ of 458 in the early supplementation group and $22(4.9 \%)$ of 446 in the delayed supplementation group (risk difference $=0.74 \%, 95 \% \mathrm{CI}=-2.2 \%$ to $3.7 \%$ and $99 \% \mathrm{CI}=-3.1 \%$ to $4.6 \%, P=0.61$ ). Results were similar when the number of tablets of folic acid taken was taken into account. In the subgroup of women who received three tablets per day, the prevalence of parasitemia on day 14 was $6.5 \%$ in the early folic acid supplementation group and $8.4 \%$ in the delayed supplementation group. Parasite clearance rates were similar in the two groups. Among women who were parasitemic at enrollment, 116 (91\%) of 128 women in the early supplementation group no longer had

TABLE 1

Baseline characteristics of the women at enrollment

\begin{tabular}{|c|c|c|}
\hline & $\begin{array}{l}\text { Early folic acid } \\
\text { supplementation } \\
(\mathrm{n}=522)\end{array}$ & $\begin{array}{l}\text { Late folic acid } \\
\text { supplementation } \\
(\mathrm{n}=513)\end{array}$ \\
\hline \multicolumn{3}{|l|}{ Age (years) } \\
\hline $15-19$ & $299(61 \%)$ & $298(60 \%)$ \\
\hline $20-29$ & $190(39 \%)$ & $188(38 \%)$ \\
\hline$\geq 30$ & $1(0.2 \%)$ & $4(0.8 \%)$ \\
\hline \multicolumn{3}{|l|}{$\begin{array}{l}\text { Education, highest level } \\
\text { attained }\end{array}$} \\
\hline No formal education & $54(10 \%)$ & $45(9 \%)$ \\
\hline Madrassa (Islamic & & \\
\hline school) & $366(70 \%)$ & $364(71 \%)$ \\
\hline Primary & $40(8 \%)$ & $38(7 \%)$ \\
\hline Secondary or higher & $61(12 \%)$ & $65(13 \%)$ \\
\hline \multicolumn{3}{|l|}{ Ethnic group } \\
\hline Mandinka & 244 (47\%) & $235(46 \%)$ \\
\hline Fula & $127(24 \%)$ & $133(26 \%)$ \\
\hline Wolof & $114(22 \%)$ & $115(23 \%)$ \\
\hline Other & $37(7 \%)$ & $28(5 \%)$ \\
\hline \multicolumn{3}{|l|}{$\begin{array}{l}\text { Mean hemoglobin at } \\
\text { enrollment (range) }\end{array}$} \\
\hline \multicolumn{3}{|l|}{ Plasmodium falciparum } \\
\hline stages) & $144 / 520(28 \%)$ & $150 / 510(29 \%)$ \\
\hline
\end{tabular}

asexual stage $P$. falciparum parasitemia at day 14 compared with 122 (92\%) of 133 women in the delayed supplementation group, which is a difference of $1.1 \%(95 \% \mathrm{CI}=-5.6 \%$ to $8.0 \%, P=0.75)$. Among women who received three tablets per day, the percentages were $88 \%$ and $91 \%$, respectively. Season did not influence the interaction between SP and folic acid.

Effect of folic acid supplementation on $\mathrm{Hb}$ concentration. Hemoglobin concentration was measured on days 0 and 14 in 886 women. On presentation, $\mathrm{Hb}$ concentrations in women in the early and delayed supplementation groups were $9.68 \mathrm{~g} / \mathrm{dL}$ and $9.61 \mathrm{~g} / \mathrm{dL}$, respectively. However, at day 14, women in the early folic acid supplementation group had a slightly higher mean $\mathrm{Hb}$ concentration than women in the delayed supplementation group $(9.76 \mathrm{~g} / \mathrm{dL}$ versus $9.68 \mathrm{~g} / \mathrm{dL})$. Adjusting for differences in the baseline $\mathrm{Hb}$ concentration, the early supplementation group still had a slightly higher mean $\mathrm{Hb}$ concentration, a difference of $0.14 \mathrm{~g} / \mathrm{dL}$ ( $95 \% \mathrm{CI}=$ $0.01-0.270, P=0.04)$. The difference in $\mathrm{Hb}$ concentration was slightly greater among women who received higher dose of folic acid but this interaction was not statistically significant.

\section{DISCUSSION}

Although SP and folic acid are now given concurrently to pregnant women in clinics across Africa, it is not known whether co-administration of folic acid interferes with the anti-malarial activity of SP when used for IPTp. We are not aware of any previous study that has addressed this issue directly. On the Thailand/Myanmar border, recent prior supplementation with iron and folic acid was a risk factor for an episode of $P$. vivax malaria but not for $P$. falciparum infections. ${ }^{10}$

A small number of studies have looked for evidence of an interaction between folic acid and SP when these drugs have been used together in the management of children with acute malaria. In a study undertaken in The Gambia, the parasite failure rate at day 14 after treatment with SP was higher in children who had received concurrent treatment with folic acid than in those who had not. ${ }^{11}$ In this study, a relatively large dose of $5 \mathrm{mg}$ of folic acid per day was given. In Kenya, subjects with clinical malaria (mean age $=80$ months) who received $2.5-5 \mathrm{mg}$ of folic acid at the time of treatment with SP cleared parasites more slowly than patients in the control group during the first week after treatment, but subsequently the rate of parasite clearance was similar in the two groups. Folic acid treatment did not effect the clinical cure rate. ${ }^{12}$ Similar results were obtained in Zambia. In this study of children with acute malaria treated with SP, the prevalence of parasitemia was significantly higher in those who had daily supplementation with $1 \mathrm{mg}$ of folic acid per day for 14 days than in those who received placebo at day 3 after the start of treatment but not at days 14 or 28 (Mulenga M, 2003. A randomised controlled trial of atovaquone/proguanil versus sulfadoxine/pyrimethamine, with or without folic acid supplementation, in the treatment of severe Plasmodium falciparum malarial anaemia. PhD thesis. University of London, London, United Kingdom). The results of these studies indicate that, in children, administration of folic acid at the time of treatment with SP does have some inhibitory effect on parasite clearance, which is most marked in the first week after treatment. 
We did not find any reduction in the parasitologic cure rate in primigravidae who received folic acid supplementation together with SP. There are a number of possible reasons for this finding. First, parasite prevalence was measured only at day 14 after treatment and not earlier as in the pediatric studies, the results of which were not known at the time that this trial was started. Thus, an early delay in parasite clearance may have been missed. Second, the dose of folic acid received by the primigravidae in this study $(500-1,500 \mu \mathrm{g} /$ day) was proportionally much less than that used in the pediatric studies and may have produced smaller increases in plasma and red blood cell folate concentrations. Finally, the sample size was relatively small and a small effect of folic acid could have been missed. However, the study was adequately powered to rule out anything more than a $5 \%$ increase in parasite prevalence at day 14 after the start of treatment.

The study was conducted in an area where the prevalence of $P$. falciparum resistance to SP is moderate. This is indicated by the fact that the day 14 parasitologic cure in the study women was greater than $90 \%$ and by the results of a trial conducted in children with clinical malaria in the same area in the year prior to the current study (Dunyo $S$ and others, unpublished data). In this pediatric trial, the prevalence of parasitologic failure at day 28 after treatment with SP was $6 \%$ after correction by polymerase chain reaction for reinfections and $18 \%$ overall. The prevalence in pre-treatment samples of the triple mutations in the dihydrofolate reductase gene (positions 51, 59, and 108) associated with resistance to pyrimethamine was $67 \%$ and that of the mutation at position 437 in the dihydropteroate synthetase gene associated with resistance to sulfonamides was $49 \%$. In many areas of east Africa, levels of resistance to SP are much higher than found in the Farafenni study area, and it is possible that in such communities the inhibitory effect of folic acid supplementation on the effectiveness of SP might be more marked. Isolates of $P$. falciparum vary in their ability to use exogenous folate and parasites with a higher propensity to do this might be able to make better use of the higher plasma concentrations provided through folic acid supplementation. ${ }^{13}$ However, it is unlikely that the doses of folic acid usually used in pregnancy would have a major effect on plasma or red blood cell folate concentrations.

Supplementation with folic acid is given widely in antenatal clinics throughout Africa to prevent anemia, but there is limited evidence of its efficacy in improving the $\mathrm{Hb}$ concentration when used in this way, in contrast to the impact of iron. There appear to be major geographic differences within Africa in the importance of folic acid deficiency as a cause of anemia in pregnancy. In western Nigeria it is a major cause, ${ }^{14}$ but in coastal Kenya, only a few primigravidae had subnormal folate levels. ${ }^{15}$ However, assessment of folate deficiency in areas where malaria is prevalent is complicated by the fact that malaria increases red blood cell folate concentration, perhaps by increasing the proportion of circulating reticulocytes that contain a higher concentration of folate than mature red blood cells. ${ }^{15}$ Folic acid did not increase the $\mathrm{Hb}$ concentration in supplementation studies in Uganda and Nigeria, in contrast to anti-malarial drugs. ${ }^{16,17}$ Thus, we were surprised to find a small but statistically significant difference in $\mathrm{Hb}$ concentrations between women who took folic acid supplementation for 14 days compared with those who did not. This suggested that some were folate deficient and had benefited from this intervention. However, since the difference between groups was small, it may have been a chance finding and it is unlikely to be clinically important.

Overall, the results of this study are reassuring. Folic acid supplementation did not increase the risk of parasitologic failure in asymptomatic primigravidae given IPTp with SP. However, these results need confirmation in an area where the prevalence of resistance to SP is higher than in The Gambia, and the effect of folic acid on the rate of clearance of parasites by women given SP merits further study.

Received August 19, 2005. Accepted for publication February 23, 2006.

Acknowledgments: We thank the women who agreed to participate in this study. We also thank Sana Jawara (Divisional Health Office, Lower River Division); field supervisors Fabakaray Sanyang, Momodou Jobe, and Faramba Ceesay; and the many field assistants and laboratory staff who contributed to the trial; the members of the DSMB (Dr. Aggrey Oloo, Professor B. J. Brabin, Dr. K. Bojang, and Dr. D. M. Schellenberg) for their help with the project; and Dr. Harparkash Kaur for undertaking the high-performance liquid chromatography analyses. The support of Margaret Pinder in the supervision of laboratory staff and the support of the administrative staff of the MRC Laboratories (Banjul, The Gambia) is gratefully acknowledged.

Financial support: This study was supported by the Medical Research Council and the Gates Malaria Partnership, which receives funding from the Bill \& Melinda Gates Foundation.

Authors' addresses: Amadou Mbaye, Sam Dunyo, and Gijs Walraven, Medical Research Council Laboratories, Banjul, The Gambia. Keshena Richardson, Caroline Shulman, Paul Milligan, and Brian Greenwood, Department of Infectious and Tropical Diseases. London School of Hygiene and Tropical Medicine, Keppel Street, London WC1E 7HT, United Kingdom. Baba Balajo, Department of Health, The Government of The Gambia, Banjul, The Gambia.

\section{REFERENCES}

1. Brabin BJ, 1991. The risk and severity of malaria in pregnant women. Applied Field Research in Malaria Reports 1. Geneva: World Health Organization.

2. Menendez C, 1995. Malaria during pregnancy: a priority area of malaria research and control. Parasitol Today 2: 178-183.

3. Guyatt HL, Snow RW, 2001. Malaria in pregnancy as an indirect cause of infant mortality in sub-Saharan Africa. Trans $R$ Soc Trop Med Hyg 95: 569-576.

4. ter Kuile FO, Terlouw DJ, Phillips-Howard PA, Hawley WA, Friedman JF, Kariuki SK, Shi YP, Kolczak MS, Lal AA, Vulule JM, Nahlen BL, 2003. Reduction of malaria during pregnancy by permethrin-treated bed nets in an area of intense perennial malaria transmission in Western Kenya. Am J Trop Med Hyg 68 (Suppl 4): 50-60.

5. Schultz LJ, Steketee RW, Macheso A, Kazembe P, Chitsulo I, Wirima JJ, 1994. The efficacy of antimalarial regimens containing sulfadoxine-pyrimethamine and/or chloroquine in preventing peripheral and placental Plasmodium falciparum infection amongst pregnant women in Malawi. Am J Trop Med Hyg 51: 515-522.

6. Parise ME, Ayisi JG, Nahlen BL, Schultz LJ, Roberts JM, Misore A, Muga R, Oloo AJ, Steketee RW, 1998. Efficacy of sulfadoxine pyrimethamine for prevention of placental malaria in an area of Kenya with a high prevalence of malaria and human immunodeficiency virus infection. Am J Trop Med Hyg 59: 813-822.

7. Shulman CE, Dorman EK, Cutts F, Kawuondo K, Bulmer JN, Peshu N, Marsh K, 1998. Intermittent sulphadoxinepyrimethamine to prevent severe anaemia secondary to malaria in pregnancy: a randomised placebo-controlled trial. Lancet 353: 632-636.

8. Rogerson SJ, Chaluluka E, Kanjala M, Mkundika P, Mhango C, 
Molyneux ME, 2000. Intermittent sulfadoxine-pyrimethamine in pregnancy: effectiveness against malaria morbidity in Blantyre, Malawi, in 1997-1999. Trans $R$ Soc Trop Med Hyg 94: 549-553.

9. Greenwood BM, Bradley AK, Greenwood AM, Byass P, Jammeh K, Marsh K, Tulloch S, Oldfield FSJ, Hayes R, 1987. Mortality and morbidity from malaria among children in a rural area of the Gambia, West Africa. Trans R Soc Trop Med Hyg 81: 478-486.

10. Nacher M, McGready R, Stepniewska K, Cho T, Looareesuwan S, White NJ, Nosten F, 2003. Haematinic treatment of anaemia increases the risk of Plasmodium vivax malaria in pregnancy. Trans R Soc Trop Med Hyg 97: 273-276.

11. Boele van Hensbroek M, Morris-Jones S, Meisner S, Jaffar SB, Dackour R, Phillips C, Greenwood BM, 1995. Iron, but not folic acid, combined with effective antimalarial therapy promotes haematological recovery in African children with acute falciparum malaria. Trans $R$ Soc Trop Med Hyg 89: 672-676.

12. Carter JY, Loolpapit MP, Lema OE, Tome JL, Nagelkerke NJD, Watkins WM, 2005. Folic acid supplementation reduces the efficacy of antifolate antimalarial therapy. Am J Trop Med Hyg 73: 166-170.

13. Hyde JE, 2005. Exploring the folate pathway in Plasmodium falciparum. Acta Trop 94: 191-206.

14. Fleming AF, 1989. Tropical obstetrics and gynaecology. 1. Anaemia in pregnancy in tropical Africa. Trans $R$ Soc Trop Med Hyg 83: 441-448.

15. Shulman CE, Graham WJ, Jilo H, Lowe BS, New L, Obiero J, Snow RW, Marsh K, 1996. Malaria is an important cause of anaemia in primigravidae: evidence from a district hospital in Kenya. Trans $R$ Soc Trop Med Hyg 90: 535-539.

16. Hamilton PJS, Gebbie DAM, Wilks NE, Lothe F, 1972. The role of malaria, folic acid deficiency and haemoglobin AS in pregnancy at Mulago Hospital. Trans $R$ Soc Trop Med Hyg 66: 594-602.

17. Fleming AF, Ghatoura GBS, Harrison KA, Briggs ND, Dunn DT, 1986. The prevention of anaemia in pregnancy in primigravidae in the guineas savanna of Nigeria. Ann Trop Med Parasitol 80: 211-233. 